\title{
The effect of midazolam on conscious, controlled processing: Evidence from the process-dissociation procedure
}

\author{
ELLIOT HIRSHMAN \\ George Washington University, Washington, D.C. \\ JULIA FISHER \\ University of Colorado, Denver, Colorado \\ THOMAS HENTHORN \\ University of Colorado Health Sciences Center, Denver, Colorado \\ JASON ARNDT \\ Middlebury College, Middlebury, Vermont \\ and \\ ANTHONY PASSANNANTE \\ University of North Carolina, Chapel Hill, North Carolina
}

\begin{abstract}
The benzodiazepine midazolam produces a dense anterograde amnesia. Recent findings (see, e.g., Hirshman, Passannante, \& Arndt, 2001) have demonstrated that midazolam produces larger impairments on explicit memory tests such as free recall and recognition memory than on implicit memory tests such as perceptualidentification and free association. Such findings suggest that midazolam impairs conscious, controlled memory processes. In the present experiments, we used Jacoby's $(1991,1998)$ processdissociation procedure to examine this hypothesis. Our results demonstrate that midazolam increases the production of old items on the exclusion task and reduces the production of old items on the inclusion task. Moreover, generation effects, hypothesized to arise from conscious processes, are reduced by midazolam on both tasks. Analyses using both independence and redundancy models of the processdissociation procedure confirm the conclusion that midazolam impairs conscious memory processes.
\end{abstract}

It has long been known that benzodiazepines produce anterograde amnesia on traditional memory tests (Mewaldt, Hinrichs, \& Ghoneim, 1983). Recent research (Arndt, Passannante, \& Hirshman, in press; Hirshman, Fisher, Henthorn, Arndt, \& Passannante, 2002; Hirshman, Passannante, \& Arndt, 1999, 2001; Hirshman, Passannante, \& Henzler, 1999; Polster, McCarthy, O’Sullivan, Gray, $\&$ Park, 1993) has focused on the effects of midazolam, a safe, fast-acting benzodiazepine that produces a dense, albeit temporary, anterograde amnesia.

Midazolam's use in surgical procedures performed under conscious sedation provides a model for our research on memory processes. In such procedures (see, e.g., Berman et al., 1992), midazolam is administered in conjunction with narcotic analgesics and regional or

The work presented here was supported by a grant from the National Science Foundation to the first author. The authors thank Michael Masson, Eyal Reingold, and an anonymous reviewer for helpful comments on a previous version of this article. Correspondence concerning this article should be addressed to E. Hirshman, Department of Psychology, George Washington University, Washington, DC 20052 (e-mail: ellioth@gwu.edu). local anesthetics, resulting in a sedated but conscious patient. This type of sedation allows relatively normal communication with the patient, who will frequently be amnesic for the surgical procedure. With these procedures as a model, our studies have relied on the combination of preserved higher level cognitive functioning and amnesia that accompanies administration of midazolam. Thus we can examine performance on various cognitive tasks in the context of anterograde amnesia.

Midazolam's pharmacokinetics make it especially well suited for the investigation of memory processes. Because midazolam is water soluble, it is metabolized substantially faster than other benzodiazepines such as diazepam and lorazepam (Stoelting, 1991). This metabolism ensures a rapid onset of amnesia, allowing a short (e.g., 5-min) interval between the administration of midazolam and the study episode. Similarly, midazolam's metabolism allows one to examine test performance at relatively brief retention intervals (e.g., $1 \mathrm{~h}$ ) with the assurance that baseline performance levels will be similar in the midazolam and control conditions.

Midazolam's water solubility also minimizes the potential side effects of intravenous administration. Intra- 
venous injection of midazolam, in contrast to intravenous injection of non-water-soluble benzodiazepines, is painless and unlikely to cause venous irritation or thrombophlebitis (Stoelting, 1991). These safety considerations are critical to the use of midazolam in the large nonclinical samples used in memory research.

Hirshman et al. (2001) provide a comprehensive review of midazolam's cognitive effects. Here, we briefly mention three properties that motivate midazolam's use in the exploration of memory processes. First, contrary to intuition, midazolam amnesia is largely independent of sedation (Veselis, Reinsel, Feshchenko, \& Wronski, 1997). Second, midazolam amnesia arises from the disruption of encoding/consolidation processes, not retrieval processes (Polster et al., 1993). Third, midazolam amnesia is not a state-dependent effect (Polster et al., 1993).

In our research, we have explored the possibility that midazolam might specifically impair conscious, controlled memory processes. We have examined this issue by determining whether midazolam has pronounced effects on memory tests that are hypothesized to rely heavily on conscious processes. For example, Hirshman et al. (2001) have demonstrated that midazolam administered prior to the study period substantially diminished generation effects on tests of free and cued recall, but had no detectable effect on generation effects found on cued perceptual identification (cf. Toth, Reingold, \& Jacoby, 1994). Although such results are suggestive, Jacoby (1991) has criticized such task-dissociation studies, noting that individual memory tests may reflect both conscious and unconscious processes.

The purpose of this article is to use Jacoby's (1991, 1998) process-dissociation procedure to explore the hypothesis that midazolam impairs conscious processes. The process-dissociation procedure relies on results derived from exclusion and inclusion tasks that differ primarily in the instructions given to participants. Instructions in the inclusion task ask participants to retrieve a studied word in response to a test cue. If they cannot recall a studied word, participants are asked to give the first word that comes to mind when presented with the cue. In the exclusion task, participants are told to retrieve a studied word in response to the cue, but not to produce the studied word. Rather, they are told to give a word different from the studied word. (See Jacoby [1998] for extensive discussion of the importance of using these specific "direct retrieval" instructions.)

Jacoby $(1991,1998)$ hypothesizes that conscious and unconscious memory influence performance on these tasks in different ways. In the inclusion task, conscious and unconscious memory are assumed to increase the probability of producing a studied item. In the exclusion task, conscious and unconscious memory are assumed to act in opposition to each other. Unconscious memory is assumed to increase the probability of producing a studied item, whereas conscious memory is assumed to decrease the probability of producing a studied item. To provide quantitative estimates of the influence of conscious $(C)$ and unconscious $(U)$ memory, Jacoby and his colleagues have proposed that two process-dissociation equations can be applied to the results from the inclusion and exclusion tasks:

$$
\begin{aligned}
& I=C+U-(C * U), \\
& E=U *(1-C),
\end{aligned}
$$

where $I$ represents inclusion performance and $E$ represents exclusion performance. Given these equations, one can use performance in the inclusion and exclusion tasks to algebraically solve for estimates of the influence of conscious and unconscious memory on performance. ${ }^{1}$

Although significant controversies (see, e.g., Curran \& Hintzman, 1995, 1997) have arisen in the interpretation of the $U$ parameter, a range of theoretical approaches (e.g., Jacoby, 1998; Joordens \& Merikle, 1993) recognize the usefulness of the process-dissociation procedure for investigating conscious processes. In these approaches, the difference between the production of old items in the inclusion and exclusion tasks is conceptualized as reflecting conscious influences (e.g., $C=I-E$ ). Increases in the production of old items on the exclusion task coupled with decreases in the production of old items on the inclusion task, therefore, reflect an impairment of conscious processes. Thus, demonstrating that midazolam increases the production of old items on the exclusion task and decreases the production of old items on the inclusion task would be consistent with the hypothesis that midazolam impairs conscious memory processes. ${ }^{2}$

\section{The Present Experiment}

Our experiment examined the effect of midazolam on the exclusion and inclusion tasks. Although manipulating instructions within subjects allows the computation of parameter estimates for each participant (Jacoby, 1998), we manipulated instructions between subjects because we were concerned that the sedation associated with midazolam might make it difficult for participants to execute the task-switching strategy that is required when instructions are manipulated within subjects.

In our experiment, each participant completed two study-test sessions (one experimental, one control). These study-test sessions were spaced 1 week apart and were conducted at the same time of day to control for any effects of this variable (see, e.g., May \& Hasher, 1998) on memory performance. In one study-test session, a $0.03-\mathrm{mg} / \mathrm{kg}$ injection of midazolam was administered $5 \mathrm{~min}$ prior to the presentation of the study list. In the other study-test session, a saline (placebo) injection was administered at the same time. Both the experimenter and the participant were blind to the nature of the injection. This dosage and time of injection were chosen because prior research (e.g., Hirshman, Passannante, \& Arndt, 1999; Hirshman et al., 2001) demonstrated that free recall, a purported index of conscious processes, was severely impaired under these conditions.

During the study period, a list of word pairs was presented. Following the retention interval, the cue words from these word pairs and the cue words from new word 
pairs were presented to participants at test. New word pairs were used to establish baseline performance levels. For each cue word, participants were asked to retrieve associated items as specified by the appropriate instructions (exclusion or inclusion). We used a conceptual, as opposed to a perceptual, memory test on the basis of the hypothesis that conscious memory processes are more likely to influence conceptual tests (see Roediger, Weldon, \& Challis [1989] for related ideas). Thus, we hoped to maximize our chances of observing effects of conscious memory processes in the saline condition, and midazolam's consequent effects thereon.

In addition to the manipulation of type of drug (midazolam vs. saline), we also manipulated encoding task (read vs. generate) during study. For half of the word pairs presented during study, both items in the pair were presented (e.g., dog-cat) and participants were asked to read these items aloud. For the remaining half of the word pairs, the cue word was presented along with a fragment of the target word (e.g., $\operatorname{dog}_{-} c_{-}{ }_{-}$). The participants were asked to read the cue word, to generate the target from the cue word and the fragment, and to say both words aloud. We manipulated encoding task because prior studies (Hirshman \& Bjork, 1988; Toth et al., 1994) suggested that generation enhances conscious memory processes. Thus, demonstrating that midazolam reduces generation effects on the exclusion and inclusion tasks would provide converging evidence for the view that midazolam impairs conscious memory processes.

\section{METHOD}

\section{Participants}

There were 32 participants in the experiment. They were from the Denver community, and they received a $\$ 100$ payment. Participants were excluded during recruitment if they were older than 35 or younger than 18; if they were currently using benzodiazepines, narcotics, or amphetamines; if their airway was in any way compromised; if they had a serious physical or mental illness (e.g., cancer, schizophrenia); if they were pregnant; if they drank more than one alcohol-containing drink per day; or if they had a history of drug abuse. The experimental protocol was approved by the Committee on the Protection of the Rights of Human Subjects of the Colorado Multiple Institution Review Board.

\section{Design and Materials}

The experiment used a $2 \times 3 \times 2$ factorial design, with type of drug (midazolam vs. saline) and encoding task (generate vs. read vs. new) as within-subjects factors and test instructions (exclusion vs. inclusion) as a between-subjects factor.

Four hundred word pairs were used. These pairs were generated from the word association norms made available by Nelson and colleagues (Nelson, McEvoy, \& Schreiber, 1998). Word pairs were selected whose average probability of forward free association was .28. As is common in norms of free association, many of the pairs were either antonyms or synonyms. In general, the word pairs did not rhyme. The 400 pairs were divided such that 200 word pairs were used in each testing session (i.e., the midazolam and saline sessions). Of the 200 word pairs used in each session, 100 were presented as old items, with 50 of these pairs being presented as read word pairs and 50 being presented as generate word pairs. The remaining 100 word pairs were used as new word pairs and were presented only at test. Materials were counterbalanced in such a way that all word pairs appeared equally often as studied and new items and in each of the experimental conditions when the design was fully counterbalanced ( 32 participants, with 16 in each test instructions condition, represented a full counterbalancing).

\section{Procedure}

Each participant was tested in two sessions. Sessions were run in the postanesthesia recovery area of the University of Colorado hospital. All equipment necessary for basic and advanced cardiopulmonary resuscitation, including a defibrillator, is available in such areas. Prior to each session, an intravenous catheter was inserted and participants were administered an injection of either $10 \mathrm{ml}$ of saline or $0.03 \mathrm{mg} / \mathrm{kg}$ of body weight of midazolam diluted to a total volume of $10 \mathrm{ml}$. Intravenous administration was used to ensure rapid action of the drug. If a participant received a midazolam injection in the first session, that participant received a saline injection in the second session and vice versa. The order of these injections was counterbalanced across subjects. Both the participant and the experimenter were blind to the nature of the injection. Participants were monitored as if they were undergoing a diagnostic procedure under conscious sedation. Arterial oxygen saturation was continuously monitored with a pulse oximeter. In addition, participants' electrocardiograms were continuously monitored and blood pressure was monitored every $15 \mathrm{~min}$. No adverse events occurred during or following the experiment.

Five minutes following the injection, a practice list of 10 word pairs was presented. These pairs were presented in blocks of 5 read and 5 generate pairs. The order of these blocks matched the order of the read and generate blocks used in the study list for a given participant. The purpose of this practice list was to ensure that participants could carry out the read and generate study tasks. Following presentation of the practice list, read and generate word pairs were presented at study in blocks of 50 read pairs and 50 generate pairs. Block order was counterbalanced across subjects. Read and generate word pairs were presented in blocks to reduce rehearsal sharing (Slamecka \& Katsaiti, 1987).

Read pairs were presented intact in the center of the computer screen, and the participants were instructed to read each word pair aloud. Following the reading of each word pair, the experimenter pressed a key and the computer displayed the next word pair. Generate pairs were constructed by replacing the vowels of the target word in a word pair with blanks (e.g., $c a r-d r \_v \_r$ ). Pilot testing ensured that all generate targets could be completed with $95 \%$ or greater accuracy. The participants were instructed to identify the second word in the generate pair and then to say the word pair aloud. As with the reading task, the experimenter pressed a key following the generation task and the computer displayed the next word pair.

Following the study period, the participants engaged in distractor activity, including category generation tasks, digit span tasks, word searches, and perceptual puzzles, for $70 \mathrm{~min}$. The purpose of this retention interval was to minimize the effect of midazolam on baseline performance in the exclusion and inclusion tasks. (See Hirshman, Passannante, \& Arndt, 1999, 2001, for evidence that the present dosage of midazolam does not influence performance on some perception and semantic memory tasks at the end of the present retention interval.)

Following the retention interval, the participants received the exclusion task or the inclusion task. In the exclusion task, the participants were instructed (Jacoby, 1998) that they would be presented with a series of cues on the computer monitor-some from the study list, and some that could be new. Their task was to use each cue word to try to recall a target word that appeared with the cue in the study list. The participants were instructed that if they recalled a target, they were not to say the recalled target aloud. Rather, they were told to use the cue to generate an additional word and to say that word aloud. Examples were given to illustrate the process of excluding a retrieved target word. The participants were also in- 
structed that in some cases (e.g., new items), they would not be able to recall a target word to a presented cue. They were informed that this would certainly occur for some cue words because some of the cue words had not been presented during the study list. When this occurred, participants were to say the first word that came to mind when they were presented with the cue. (It is important to note that this last component of the procedure made it very unlikely that participants would simply generate words that were unrelated to the cue in the exclusion task.)

In the inclusion task, the participants were instructed (Jacoby, 1998) that they would be presented with a series of cues on the computer monitor-some from the study list and some that could be new. Their task was to use each cue word to try to recall a target word that appeared with the cue in the study list. The participants were instructed that if they recalled a target, they were to say the recalled target aloud. The participants were also instructed that in some cases (e.g., new items), they would not be able to recall a target word to a presented cue. They were informed that this would certainly occur for some cue words because some of the cue words had not been presented during the study list. When this occurred, the participants were to say the first word that came to mind when they were presented with the cue.

Following these instructions, the exclusion or inclusion task proceeded. Each cue word was presented in the center of the computer monitor and the participant responded. Following the participant's response, the experimenter pressed the space bar and the next cue appeared on the monitor. The order of test cues was random. All responses were recorded on audiotape and were later transcribed by the experimenter for scoring.

At the conclusion of each session, all participants were discharged as if they had received midazolam. They received a ride home and were instructed not to drive until the next day. All participants were debriefed following their second session.

\section{RESULTS}

Here we will describe exclusion performance followed by inclusion performance. In each case, we will first discuss the proportions of items read and generated correctly during the study period. We will then turn to the results from the memory task.

\section{Exclusion Condition}

In the exclusion condition, the proportion of items correctly identified at study was above .97 in all conditions (read-saline $=1.00$, generate-saline $=.99$, readmidazolam $=.99$, and generate-midazolam $=.98$ ). Thus, participants were able to carry out the tasks of reading word pairs and generating responses even when injected with the dose of $0.03 \mathrm{mg} / \mathrm{kg}$ of midazolam.

The mean proportions of items produced at test in the exclusion task are presented in the top panel of Table 1 as a function of type of drug (midazolam vs. saline) and encoding condition (generate vs. read vs. new). These data display two important results. First, the production of old items (generate and read) increased in the midazolam condition. In the saline condition, the production of old items was lower than baseline performance, whereas in the midazolam condition, the production of old items equaled baseline performance. Second, production of generate items was lower than production of read items in the saline condition, but this generation effect did not occur in the midazolam condition.
Table 1

Mean Proportion of Items Produced (With Standard Deviations) in the Exclusion and Inclusion Tasks as a Function of Type of Drug (Midazolam vs. Saline) and Encoding Condition (Read vs. Generate vs. New)

\begin{tabular}{|c|c|c|c|c|c|c|}
\hline \multirow[b]{2}{*}{ Type of Drug } & \multicolumn{2}{|c|}{ Generate } & \multicolumn{2}{|c|}{ Read } & \multicolumn{2}{|c|}{ New } \\
\hline & $M$ & $S D$ & $M$ & $S D$ & $M$ & $S D$ \\
\hline \multicolumn{7}{|c|}{ Exclusion } \\
\hline Saline & .14 & .06 & .19 & .07 & .24 & .07 \\
\hline Midazolam & .24 & .09 & .24 & .09 & .25 & .08 \\
\hline \multicolumn{7}{|c|}{ Inclusion } \\
\hline Saline & .62 & .13 & .50 & .12 & .31 & .06 \\
\hline Midazolam & .45 & .12 & .37 & .12 & .27 & .07 \\
\hline
\end{tabular}

A $3 \times 2$ within-subjects analysis of variance (ANOVA) conducted on the exclusion responses using the factors of encoding task (generate vs. read vs. new) and type of drug (midazolam vs. saline) produced results consistent with the preceding conclusions. There were three significant effects. First, the interaction of encoding task and type of drug was significant $\left[F(2,30)=3.53, M S_{\mathrm{e}}=\right.$ $0.005, p<.05]$. In addition, the main effects of type of drug $\left[F(1,15)=9.34, M S_{\mathrm{e}}=0.009, p<.01 ;\right.$ midazolam $=.24$, saline $=.19]$ and of encoding task $[F(2,30)=$ $8.66, M S_{\mathrm{e}}=0.003, p<.005 ;$ generate $=.19$, read $=.21$, new $=.25]$ were significant.

To explore the interaction of type of drug and encoding task, we compared performance on old (i.e., generate and read) and new items in the midazolam and saline conditions. Production of old items in the saline condition was significantly lower than production of old items in the midazolam condition $\left[F(1,15)=12.26, M S_{\mathrm{e}}=\right.$ $0.008, p<.003$ ]. In contrast, production of new items was approximately equal in the midazolam and saline conditions $(p>.5)$. Examination of the generation effect in the saline and midazolam conditions demonstrated that production of generate items was lower than production of read items in the saline condition $[F(1,15)=$ $9.36, p<.01]$, whereas performance in these conditions was approximately equal in the midazolam condition $(p>.5)$.

\section{Inclusion Condition}

In the inclusion condition, the proportion of items correctly identified at study was above .96 in all conditions (read-saline $=1.00$, generate-saline $=.98$, readmidazolam $=.99$, and generate-midazolam $=.97$ ). Thus, participants were able to carry out the tasks of reading word pairs and generating responses even when injected with the dose of $0.03 \mathrm{mg} / \mathrm{kg}$ of midazolam.

The mean proportions of items produced in the inclusion task are presented in the bottom panel of Table 1 as a function of type of drug (midazolam vs. saline) and encoding condition (generate vs. read vs. new). These data display two important results. First, performance on old items (generate and read) was lower in the midazolam condition than in the saline condition on the inclusion 
task. Second, production of generate items was greater than production of read items in the saline condition, and this effect diminished in the midazolam condition. Interestingly, and in contrast to the exclusion task, there was a residual generation effect in the midazolam condition on the inclusion task.

A $3 \times 2$ within-subjects ANOVA conducted on the inclusion responses using the factors of encoding task (generate vs. read vs. new) and type of drug (midazolam vs. saline) produced results consistent with the preceding conclusions. There were three significant effects. The interaction of encoding task and type of drug was significant $\left[F(2,30)=10.51, M S_{\mathrm{e}}=0.003, p<.001\right]$. In addition, the main effects of type of drug $\left[F(1,15)=62.27, M S_{\mathrm{e}}=\right.$ $0.005, p<.001 ;$ saline $=.48$, midazolam $=.36]$ and encoding task $\left[F(2,30)=61.37, M S_{\mathrm{e}}=0.008, p<.001\right.$; generate $=.54$, read $=.44$, new $=.29]$ were significant.

To explore the interaction of type of drug and encoding task further, we compared performance on old and new items across the saline and midazolam conditions.

Production of old items in the saline condition was significantly greater than production of old items in the midazolam condition $\left[F(1,15)=60.24, M S_{\mathrm{e}}=0.001, p<\right.$ $.001]$. Importantly, production of new items was also significantly greater in the saline than in the midazolam condition $\left[F(1,15)=8.64, M S_{\mathrm{e}}=0.002, p<.05 ;\right.$ saline $=$ .31 , midazolam $=.27]$. Even though the numerical difference in baseline performance was small, we used multiple procedures for correcting for baseline differences (e.g., threshold correction, $d^{\prime}, A^{\prime}$ ) to reexamine the comparison of old performance in the saline and midazolam conditions. Not surprisingly, given the small baseline difference and the robustness of the reported effect, all measures demonstrated greater production of old items in the saline condition.

Examination of the generation effect in the saline and midazolam conditions demonstrated that production of generate items was greater than production of read items in both conditions $[F(1,15)=16.02, p<.005$ for the saline condition, and $F(1,15)=14.23, p<.005$ for the midazolam condition]. Thus, even though the interaction of encoding condition and type of drug reflects a numerically larger generation effect in the saline condition, there was a residual generation effect in the midazolam condition.

\section{DISCUSSION}

Our primary finding is that midazolam produced dissociative effects on exclusion and inclusion task performance. Midazolam increased the production of old items in the exclusion task and decreased the production of old items in the inclusion task. Moreover, midazolam reduced generation effects in both tasks. As outlined in the introduction, these results are consistent with the view that midazolam impairs conscious memory processes. Conscious processes are hypothesized to decrease the production of old items on the exclusion task; midazolam increased the production of old items on this task.
Similarly, conscious processes are hypothesized to increase the production of old items on the inclusion task; midazolam decreased the production of old items on this task. Furthermore, generation effects are hypothesized to depend on conscious processes and midazolam decreased generation effects on both tasks. Together with our prior results (e.g., Hirshman et al., 2001), these results provide strong converging evidence that midazolam impairs conscious memory processes.

Although our between-subjects manipulation of test instructions did not permit statistical tests of the $C$ parameter, it is useful to verify the preceding conclusion by examining the ordering of the $C$ parameter across experimental conditions. To accomplish this, we examined reparameterizations of our mean results produced by Jacoby's (1991) independence model, Joordens and Merikle's (1993) redundancy model, and variants of each of these models that used Yonelinas, Regehr, and Jacoby's (1995) procedures for incorporating new performance. ${ }^{3}$ We examined both independence and redundancy models to ensure that our conclusions were independent of the assumptions one makes about the stochastic relation between conscious and unconscious processing. We examined models incorporating Yonelinas et al.'s procedure to ensure that our conclusions did not depend on the exclusion of baseline performance. Furthermore, although we chose Yonelinas et al.'s procedure because of its mathematical tractability and prior success, alternative correction procedures (e.g., a simple threshold model) produce similar results for estimates of $C$. For each model, there are parameters representing conscious $(C)$ and unconscious $(U)$ processes. In addition, there is a criterion parameter for each task (inclusion and exclusion) in the models that incorporate baseline performance.

The results of the re-parameterizations are presented in Table 2. The most important result shown is that midazolam substantially decreases $C$ in all models. Moreover, there is a generation effect on $C$ in the saline condition and midazolam reduces the size of this effect in all models. Thus, all of the models confirm all of our conclusions.

Although our focus has been on conscious processes in this paper, the results presented in Table 2 raise two questions about unconscious processes. First, one can ask whether midazolam impairs unconscious, as well as conscious, processing. Demonstrating that midazolam has limited effects on estimates of $U$ would be consistent with the view that midazolam primarily affects conscious processes. Second, one can also examine the effect of generation on unconscious processes. In a prior study using the process-dissociation procedure to explore stem completion performance, Toth et al. (1994) demonstrated that generation increased $C$, but decreased $U$. Our study provides an opportunity to examine whether this negative generation effect replicates in a conceptual word association test.

Unfortunately, the results presented in Table 2 are ambiguous with respect to effects on $U$. Midazolam diminishes $U$ under the redundancy models, but this does not 
Table 2

Parameter Estimates for $C, U, C R_{i}$, and $C R_{e}$ in the Independence, Redundancy, and Corresponding Baseline Models as a Function of Type of Drug (Saline vs. Midazolam) and Encoding Condition (Generate vs. Read vs. New)

\begin{tabular}{|c|c|c|c|c|c|c|c|c|c|}
\hline \multirow[b]{2}{*}{ Model } & \multirow[b]{2}{*}{ Condition } & \multicolumn{2}{|c|}{$C$} & \multicolumn{2}{|c|}{$U$} & \multicolumn{2}{|c|}{$C R_{i}$} & \multicolumn{2}{|c|}{$C R_{e}$} \\
\hline & & Gen. & Read & Gen. & Read & Gen. & Read & Gen. & Read \\
\hline \multirow[t]{2}{*}{ Independence } & Saline & .48 & .31 & .27 & .28 & & & & \\
\hline & Midazolam & .21 & .13 & .30 & .28 & & & & \\
\hline \multirow[t]{2}{*}{ Independence baseline } & Saline & .44 & .26 & .03 & .05 & -.50 & -.50 & -.71 & -.71 \\
\hline & Midazolam & .19 & .11 & .14 & .06 & -.61 & -.61 & -.67 & -.67 \\
\hline \multirow[t]{2}{*}{ Redundancy } & Saline & .48 & .31 & .62 & .50 & & & & \\
\hline & Midazolam & .21 & .13 & .45 & .37 & & & & \\
\hline \multirow[t]{2}{*}{ Redundancy baseline } & Saline & .74 & .54 & .80 & .49 & -.50 & -.50 & -.71 & -.71 \\
\hline & Midazolam & .44 & .31 & .49 & .28 & -.61 & -.61 & -.67 & -.67 \\
\hline
\end{tabular}

Note- $C$ and $U$ are parameters for conscious and unconscious processes; $C R_{i}$ and $C R_{\rho}$ are the criterion parameters for inclusion and exclusion tasks. For futher explanation, see the Discussion.

occur under the independence models. Similarly, there is a generation effect on $U$ under the redundancy models, but this effect does not occur consistently under the independence models. Given that our study was not designed to discriminate between the redundancy and independence models, we must remain agnostic regarding the implications of the present results for midazolam's and generation's effects on unconscious processing.

In the context of considering alternative interpretations, we should also raise the question of whether the present results demand a dual-process interpretation. Dunn and Kirsner (1988) have argued that the rejection of a single-process model requires the demonstration of a nonmonotonic relation between performance on two tasks. Figure 1 presents performance in the exclusion task plotted as a function of performance in the inclusion task for the factorial combinations of the encoding condition and type of drug variables. Figure 1 demonstrates that exclusion performance can be represented as a monotonic function of inclusion performance. As such, the present results do not necessarily rule out a single-process interpretation of the inclusion and exclusion tasks. Nonetheless there are two reasons why we favor a dual-process interpretation. First, and most importantly, other results obtained in the inclusion and exclusion tasks (see, e.g., Toth et al., 1994, Experiment 2) demonstrate nonmonotonic functions. These findings suggest that a dual-process interpretation is the most parsimonious account of the overall pattern of results in the literature. Second, although the form of the function in Figure 1 demonstrates monotonicity, the function contains an extended flat region (when inclusion changes from .27 to .45) and a sharp "elbow" (when inclusion changes from .45 to .50). As such, one would have to make complex assumptions about the relations between the underlying memory process and the two tasks to explain these features of Figure 1 (e.g., that the exclusion and inclusion tasks vary dramatically in their sensitivity to the effects of the underlying memory process, but then this sensitivity reverses at a threshold point). The complexity of these assumptions mitigates any parsimony advantage suggested by the single-process view.
Evidence from task-dissociation paradigms (e.g., Hirshman et al., 2001) suggests that midazolam impairs conscious, controlled memory processes. The present study has demonstrated that midazolam increases the production of old items on the exclusion task and reduces the production of old items on the inclusion tasks. Moreover, generation effects, hypothesized to arise from conscious processes, are reduced by midazolam on both tasks. These results provide compelling, converging evidence that midazolam substantially impairs conscious memory processes. These results motivate further studies designed to determine whether midazolam's amnesic effects are specific to conscious memory processes.

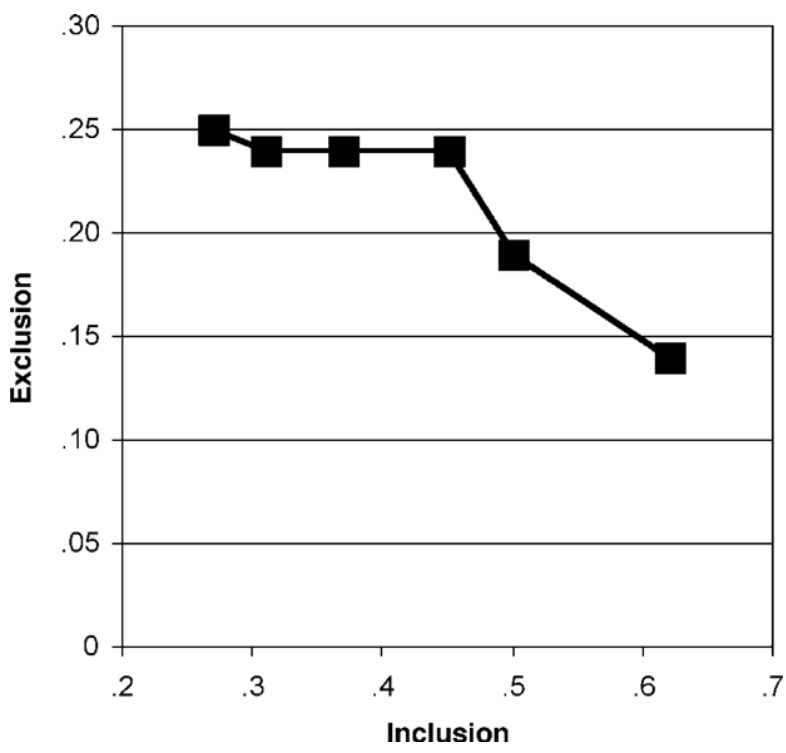

Figure 1. Proportion of items produced on exclusion and inclusion tasks for the experimental conditions resulting from the factorial combination of encoding condition (generate vs. read vs. new) and type of drug (saline vs. midazolam). Moving from left to right across the figure, the conditions are new-midazolam, new-saline, read-midazolam, generate-midazolam, read-saline, and generate-saline. 


\section{REFERENCES}

Arndt, J., Passannante, A., \& Hirshman, E. (in press). The effect of midazolam on implicit and explicit memory in category exemplar production and category cued recall. Memory.

Berman, L., Chernik, D., Cooper, S., Davidson, A., DuPen, S., et al. (1992). Reversal of central benzodiazepine effects by intravenous flumazenil after conscious sedation with midazolam and opioids: A multicenter clinical study. Clinical Therapeutics, 14, 878-894.

Buchner, A., Erdfelder, E., \& VAterrodt-Plünnecke, B. (1995). Toward unbiased measurement of conscious and unconscious memory processes within the process dissociation framework. Journal of Experimental Psychology: General, 124, 137-160.

Curran, T., \& Hintzman, D. (1995). Violations of the independence assumptions in process dissociation. Journal of Experimental Psychology: Learning, Memory, \& Cognition, 21, 531-547.

Curran, T., \& Hintzman, D. (1997). Consequences and causes of correlations in process dissociation. Journal of Experimental Psychology: Learning, Memory, \& Cognition, 23, 496-504.

DunN, J., \& KIRSNER, K. (1988). Discovering functionally independent mental processes: The principle of reversed association. Psychological Review, 95, 91-101.

Hirshman, E. (in press). Perspectives on the measurement of automatic and controlled memory processes: Ordinal inferences in the processdissociation procedure. Psychological Review.

HiRshman, E., \& BJoRK, R. (1988). The generation effect: Support for a two-factor theory. Journal of Experimental Psychology: Learning, Memory, \& Cognition, 14, 484-494.

Hirshman, E., Fisher, J., Henthorn, T., Arndt, J., \& Passannante, A. (2002). Midazolam amnesia and dual-process models of the wordfrequency mirror effect. Journal of Memory \& Language, 47, 499516.

Hirshman, E., Passannante, A., \& Arndt, J. (1999). The effect of midazolam on the modality match effect in implicit memory. Cognitive Brain Research, 7, 473-479.

Hirshman, E., Passannante, A., \& Arndt, J. (2001). Midazolam amnesia and conceptual processing in implicit memory. Journal of Experimental Psychology: General, 130, 453-465.

Hirshman, E., Passannante, A., \& Henzler, A. (1999). The influence of midazolam on implicit memory tests. Brain \& Cognition, 41, 351-364.

JACOBY, L. L. (1991). A process dissociation framework: Separating automatic from intentional uses of memory. Journal of Memory \& Language, 30, 513-541.

JACOBY, L. L. (1998). Invariance in automatic influences of memory: Toward a user's guide for the process-dissociation procedure. Journal of Experimental Psychology: Learning, Memory, \& Cognition, 24, 3-26.

Joordens, S., \& Merikle, P. (1993). Independence or redundancy? Two models of conscious and unconscious influence. Journal of Experimental Psychology: General, 122, 462-467.

MAY, C., \& HASHER, L. (1998). Synchrony effects in inhibitory control over thought and action. Journal of Experimental Psychology: Human Perception \& Performance, 24, 363-379.

Mewaldt, S. P., Hinrichs, J. V., \& Ghoneim, M. M. (1983). Diazepam and memory: Support for a duplex model of memory. Memory \& Cognition, 11, 557-564.

Nelson, D. L., McEvoy, C. L., \& Schreiber, T. A. (1998). The Uni- versity of Florida word association, rhyme, and word fragment norms. Available from http://www.usf.edu/FreeAssociation/.

Polster, M. R., McCarthy, R. A., O'Sullivan, G., Gray, P. A., \& PARK, G. R. (1993). Midazolam-induced amnesia: Implications for the implicit/explicit memory distinction. Brain \& Cognition, 22, 244-265.

Roediger, H. L., III, Weldon, M. S., \& Challis, B. (1989). Explaining dissociations between implicit and explicit measures of retention: A processing account. In H. L. Roediger III \& F. I. M. Craik (Eds.), Varieties of memory and consciousness: Essays in honour of Endel Tulving (pp. 3-41). Hillsdale, NJ: Erlbaum.

SlameCKA, N., \& KATSAiti, L. (1987). The generation effect as an artifact of selective displaced rehearsal. Journal of Memory \& Language, 26, 589-607.

Stoelting, R. K. (1991). Pharmacology and physiology in anesthetic practice (2nd ed.). Philadelphia: J. B. Lippincott.

Toth, J. P., Reingold, E. M., \& JACOBY, L. (1994). Toward a redefinition of implicit memory: Process dissociations following elaborative processing and self-generation. Journal of Experimental Psychology: Learning, Memory, \& Cognition, 20, 290-303.

Veselis, R. A., Reinsel, R. A., Feshchenko, V., \& Wronski, M. (1997). The comparative amnestic effects of midazolam, propofol, thiopental, and fentanyl at equisedative concentrations. Anesthesiology, 87, 749-764.

Yonelinas, A. P., Regehr, G., \& JACoby, L. (1995). Incorporating response bias in a dual-process theory of memory. Journal of Memory \& Language, 34, 821-835.

\section{NOTES}

1. The original version of the process dissociation equations (i.e., Equations 1 and 2) did not represent baseline performance in the inclusion and exclusion tasks. Buchner, Erdfelder, and Vaterrodt-Plünnecke (1995) and Yonelinas, Regehr, and Jacoby (1995) have presented models designed to incorporate differences in baseline performance in the inclusion and exclusion tasks.

2. In this context, Hirshman (in press) has recently proven formally that given three relatively neutral assumptions about the nature of conscious and unconscious processes, one can derive conclusions about conscious memory processes from the ordinal pattern of results on the inclusion and exclusion tasks. The assumptions are that (1) conscious and unconscious processes act in concert on the inclusion task; (2) conscious and unconscious processes act in opposition on the exclusion task, with conscious processes decreasing the production of old items; and (3) there are monotonic relations between conscious and unconscious processes and performance on the inclusion and exclusion tasks. Consistent with the algebraic representations of the independence and redundancy models, Hirshman has demonstrated that, given these assumptions, one can infer that conscious memory processes are reduced in an experimental condition if production of old items in this condition increases in the exclusion task and decreases in the inclusion task.

3. We thank Andrew Yonelinas for providing the minimization algorithm software.

(Manuscript received October 28, 2002; revision accepted for publication July 30,2003 .) 